\title{
Superhydrophobic Polyimide via Ultraviolet Photooxidation: The Evolution of Surface Morphology and Hydrophobicity under Different Ultraviolet Intensities
}

\author{
Hongyu Gu, ${ }^{1,2}$ Lixin Song, ${ }^{1}$ Jinlin Zhang, ${ }^{1}$ and Zhenyi Qi ${ }^{1}$ \\ ${ }^{1}$ Key Laboratory of Inorganic Coating Materials, Shanghai Institute of Ceramics, Chinese Academy of Sciences, \\ 1295 Dingxi Road, Shanghai 200050, China \\ ${ }^{2}$ University of Chinese Academy of Sciences, 19 Yuquan Road, Beijing 100049, China \\ Correspondence should be addressed to Lixin Song; lxsong@mail.sic.ac.cn and Zhenyi Qi; qzy@mail.sic.ac.cn
}

Received 10 October 2014; Accepted 30 November 2014

Academic Editor: Yuanlie Yu

Copyright ( 2015 Hongyu Gu et al. This is an open access article distributed under the Creative Commons Attribution License, which permits unrestricted use, distribution, and reproduction in any medium, provided the original work is properly cited.

Ultraviolet (UV) photooxidation has recently been developed to fabricate superhydrophobic polyimide (PI) films in combination with fluoroalkylsilane modification. However, it remains unclear whether the surface morphology and hydrophobicity are sensitive to technical parameters such as UV intensity and radiation environment. Herein, we focus on the effects of UV intensity on PI surface structure and wettability to gain comprehensive understanding and more effective control of this technology. Scanning electron microscopy (SEM) and atomic force microscopy (AFM) results showed that UV intensity governed the evolutionary pattern of surface morphology: lower UV intensity $\left(5 \mathrm{~mW} / \mathrm{cm}^{2}\right)$ facilitated in-plane expansion of dendritic protrusions while stronger UV $\left(10\right.$ and $\left.15 \mathrm{~mW} / \mathrm{cm}^{2}\right)$ encouraged localized growth of protrusions in a piling-up manner. Surface roughness and hydrophobicity maximized at the intensity of $10 \mathrm{~mW} / \mathrm{cm}^{2}$, as a consequence of the slowed horizontal expansion and preferred vertical growth of the protrusions when UV intensity increased. Based on these results, the mechanism that surface micro/nanostructures developed in distinct ways when exposed to different UV intensities was proposed. Though superhydrophobicity (water contact angle larger than $150^{\circ}$ ) can be achieved at UV intensity not less than $10 \mathrm{~mW} / \mathrm{cm}^{2}$, higher intensity decreased the effectiveness. Therefore, the UV photooxidation under $10 \mathrm{~mW} / \mathrm{cm}^{2}$ for $72 \mathrm{~h}$ is recommended to fabricate superhydrophobic PI films.

\section{Introduction}

Superhydrophobic materials with water contact angle (WCA) above $150^{\circ}$ have numerous potential applications such as in self-cleaning, antifrosting, anticorrosion, anti-icing, and drag reduction [1-3]. As a representative type, superhydrophobic polymers have attracted extensive interest in recent decades due to their low costs, light weight, flexibility, and ease of processing [4-6]. However, common polymers lack adequate stability against heat and tension, which seriously limits their applications in harsh environments [7]. Polyimide (PI), a widely used polymer, can avoid these drawbacks owing to its unique physical and chemical properties such as excellent thermal stability, high strength, and good chemical resistance [8-10]. Therefore, superhydrophobic PI would exhibit high durability when applied in various extreme environments (e.g., space environment) $[11,12]$.

Superhydrophobic property is influenced by both surface energy and surface roughness [1]. Generally, WCA larger than $120^{\circ}$ could only be obtained by altering the surface roughness [13]. However, PI surface is normally flat on the micron scale. Therefore, techniques including template [14-17], electrospinning $[7,18,19]$, laser $[11,20]$, and plasma [21-23] are used to tailor surface morphology for superhydrophobic PI. Unfortunately, the complex operations, novel precursors, masks, or vacuum instruments involved usually limit the industrial fabrication. A facile strategy using ultraviolet (UV) photooxidation has been put forward by our group to fabricate micro/nanostructures on originally flat PI films. Then, 
based on the proper surface morphology, superhydrophobic PI could be easily obtained by further $1 \mathrm{H}, 1 \mathrm{H}, 2 \mathrm{H}, 2 \mathrm{H}-$ perfluorodecyltriethoxysilane (FAS) treatment. Being a dry process conducted under atmospheric environment, this UV photosensitized oxidation method possesses distinctive advantages compared with other techniques. The equipment investment and operating costs are relatively low, which would largely facilitate the large-scale production. In previous work [12], we have reported the preparation procedure and detailed characterization of the superhydrophobic PI films. UV photooxidation induced surface morphology was proven to be crucial to the wettability. However, it remains unclear whether the surface morphology and hydrophobicity are sensitive to technical parameters.

In this paper, the evolution of surface morphology and corresponding hydrophobicity under different UV intensities was studied to deeply understand the effects of UV intensity and better control the hydrophobicity of PI films.

\section{Materials and Methods}

2.1. Materials. Pyromellitic dianhydride-oxydianiline-type PI films (Kapton $100 \mathrm{H}, \mathrm{C}_{22} \mathrm{H}_{10} \mathrm{O}_{5} \mathrm{~N}_{2}$ ) were obtained from Toray DuPont. $1 \mathrm{H}, 1 \mathrm{H}, 2 \mathrm{H}, 2 \mathrm{H}$-Perfluorodecyltriethoxysilane (FAS, $\left.\mathrm{C}_{10} \mathrm{~F}_{17} \mathrm{H}_{4} \mathrm{Si}\left(\mathrm{OCH}_{2} \mathrm{CH}_{3}\right)_{3}, 97 \%\right)$ was supplied by Alfa Aesar. Ultrapure water was prepared using a Milli-Q-Plus system. Analytical grade ethanol (99.7\%) was purchased from Sinopharm Chemical Reagent Co., Ltd. Prior to use, PI films were ultrasonically cleaned with purified water followed by ethanol and dried in an oven. Other chemicals were used as received.

2.2. Fabrication of Hydrophobic PI. In a typical process, the method was divided into two distinct procedures, that is, UV photooxidation and succedent fluoroalkylsilane modification (denoted by UV/FAS). UV photooxidation was conducted in air at ambient temperature in a cubic chamber $(450 \times 450 \times$ $350 \mathrm{~mm}^{2}$ ). The chamber was equipped with tubular mercuryquartz lamps $(290 \mathrm{~mm} \times 15 \mathrm{~mm}$, SunMonde) side-by-side emitting UV light at 254 and $185 \mathrm{~nm}$. The output at $185 \mathrm{~nm}$ was equivalent to about $10 \%$ of that at $254 \mathrm{~nm}$. During UV irradiation processes, rectangular PI films $\left(30 \times 40 \mathrm{~mm}^{2}\right)$ were placed at different distances (ca. $8 \mathrm{~mm}, 25 \mathrm{~mm}$, and $55 \mathrm{~mm}$ ) from the lamps, where the intensities of the $254 \mathrm{~nm}$ UV light were measured to be 5,10 , and $15 \mathrm{~mW} / \mathrm{cm}^{2}$, respectively. The light at $185 \mathrm{~nm}$ attenuated quickly in air accompanied by dissociation of oxygen molecules into oxygen atoms. Here, we denoted the intensity of $254 \mathrm{~nm}$ UV light as the intensity of UV light for convenience. At each intensity, PI films were irradiated for $24,48,72$, or $144 \mathrm{~h}$ in air at atmospheric environment. Fluoroalkylsilane modification was then carried out with FAS in heated reactors. Specifically, a few droplets $(30-50 \mu \mathrm{L})$ of FAS were dispensed at the bottom of a Teflon container $(200 \mathrm{~mL})$ using a micro-adjustable pipette $(720000$, Dragon) while the UV-treated PI films were placed vertically in the Teflon container, which was further sealed in a stainless steel autoclave. The reactor was then annealed at $120^{\circ} \mathrm{C}$ for $2 \mathrm{~h}$ to enable the vapor of FAS to react with the modified PI films.
Finally, the samples were heated at $150^{\circ} \mathrm{C}$ for $3 \mathrm{~h}$ to volatilize unreacted FAS molecules.

2.3. Characterization. The morphology and element distribution were characterized by scanning electron microscopy (SEM) coupled with energy-dispersive X-ray spectroscopy (EDS) on an FEI Magellan 400 microscope. Before the examination, the surfaces of the samples were sputter-coated with a thin layer of $\mathrm{Cd}$ to prevent charging. For the EDS mappings, the colored spots corresponded to the presence of each element. The surface roughness was analyzed using an atomic force microscope (AFM) (Nanoscope V Multimode AFM System, Bruker) in tapping mode. The average AFM value was obtained at six different positions by measuring three samples. The static WCA was measured with $5 \mu \mathrm{L}$ water and the sliding angle was tested by $10 \mu \mathrm{L}$ water using a SL200B contact angle system (Solon Tech.) at ambient temperature. The average WCA value was obtained at six different positions by measuring three samples. The sliding angle was determined by slowly tilting the sample stage until the $10 \mu \mathrm{L}$ water droplet started moving. Meanwhile, a digital video camera was employed to take photographs at the rate of 1 frame/s.

\section{Results and Discussion}

3.1. Surface Morphology. The pristine PI films possessed a morphologically flat surface and could only be rendered slight hydrophobicity $\left(\mathrm{WCA}=105.1^{\circ}\right)$ after FAS modification. Further enhancement of hydrophobicity was proven to be feasible by constructing appropriate morphology through UV irradiation [12]. Therefore, investigations of the dependence of surface morphology and roughness on the UV treatment parameters would allow for rational control of the hydrophobicity. To this end, SEM in combination with AFM was used to study the evolution of surface morphology at different UV intensities $\left(5,10\right.$, and $\left.15 \mathrm{~mW} / \mathrm{cm}^{2}\right)$. Note that samples with sequent UV exposure and FAS modification (denoted by UV/FAS-treated PI films hereafter) were used to analyze the morphological evolution, since the latter was proven to have no significant effect on the surface microstructure.

Figure 1 shows the surface microstructure of UV/FAStreated PI when UV intensity was $5 \mathrm{~mW} / \mathrm{cm}^{2}$ and irradiation period varied from $24 \mathrm{~h}$ to $144 \mathrm{~h}$. As can be seen in Figure 1(a), $24 \mathrm{~h}$ of UV irradiation resulted in uniformly distributed nanoprotrusions (in spherical or dendritic shape) and grooves on the PI surface. The protrusion areas gradually expanded and finally occupied most surface of the film as the exposure time prolonged (see Figures 1(a)-1(d)). This expansion, in fact, could be explained by the growth (in both width and length), interconnection, and final aggregation of the nanoprotrusions, as clearly presented in the enlarged SEM images (Figures $1(\mathrm{e})-1(\mathrm{~h})$ ). Expansion of the dendritic protrusions along the in-plane directions can be regarded as a main characteristic of this type of growth mode. Consequently, the initial grooves (up to 200-300 nm in size) gradually diminished and were replaced by the narrow dendritic channels (less than $100 \mathrm{~nm}$ ) in the end. 


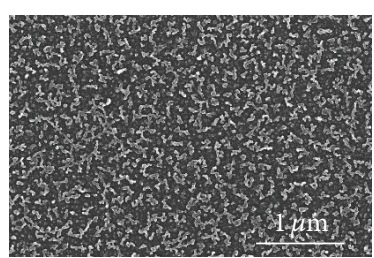

(a)

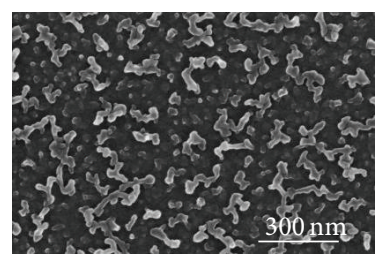

(e)

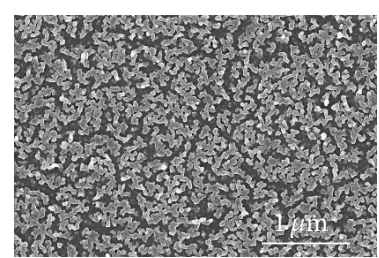

(b)

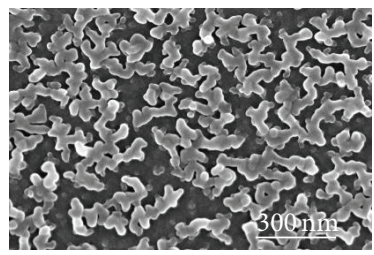

(f)

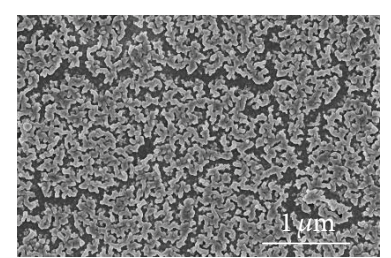

(c)

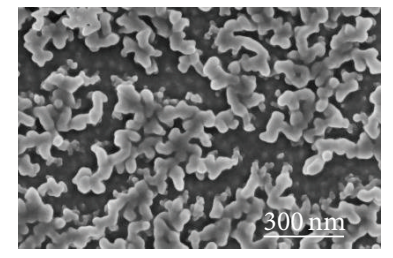

(g)

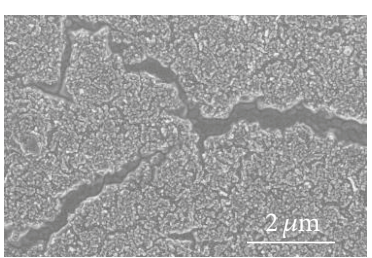

(d)

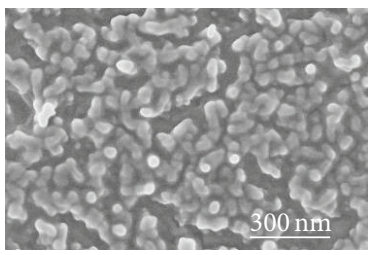

(h)

Figure 1: SEM images of the UV $\left(5 \mathrm{~mW} / \mathrm{cm}^{2}\right) /$ FAS-treated PI films. The UV exposure periods are $24 \mathrm{~h}(\mathrm{a}, \mathrm{e}), 48 \mathrm{~h}(\mathrm{~b}, \mathrm{f}), 72 \mathrm{~h}(\mathrm{c}, \mathrm{g}), \mathrm{and} 144 \mathrm{~h}$ $(\mathrm{d}, \mathrm{h})$, respectively.

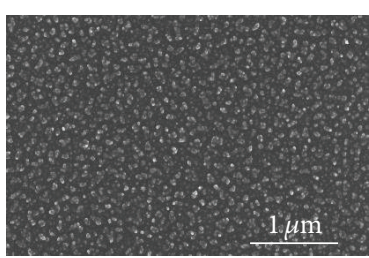

(a)

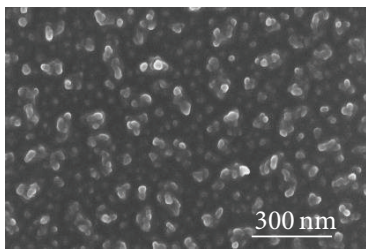

(e)

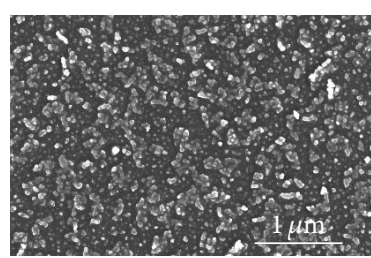

(b)

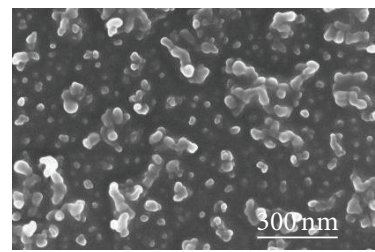

(f)

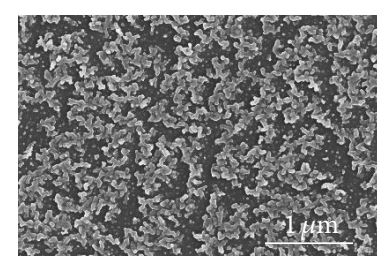

(c)

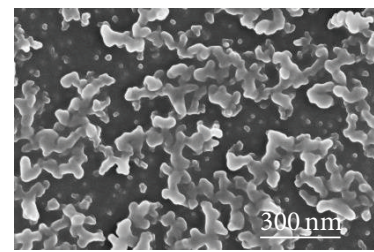

(g)

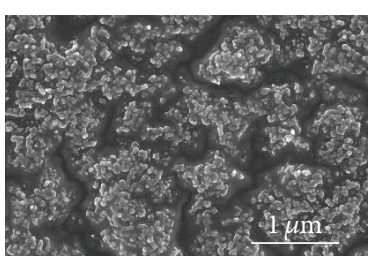

(d)

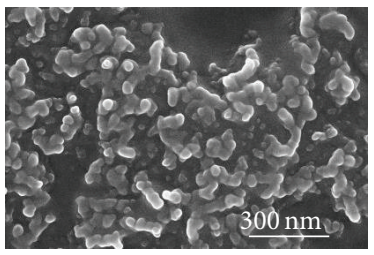

(h)

FIGURE 2: SEM images of UV $\left(10 \mathrm{~mW} / \mathrm{cm}^{2}\right) /$ FAS-treated PI films. The UV treatment periods are $24 \mathrm{~h}(\mathrm{a}, \mathrm{e}), 48 \mathrm{~h}(\mathrm{~b}, \mathrm{f}), 72 \mathrm{~h}(\mathrm{c}, \mathrm{g})$, and $144 \mathrm{~h}$ $(\mathrm{d}, \mathrm{h})$, respectively.

It should be noted that many wide grooves (up to $900 \mathrm{~nm}$ ) emerged (Figure $1(\mathrm{~d})$ ) on the $144 \mathrm{~h}$ irradiated films, separating the protrusion networks into micron-sized fragments. These wide grooves may be ascribed to the accumulated interfacial stress between the protrusions dominated surface and the underlying PI. In other words, the cracklike grooves formed as a manner to release the interfacial stress introduced by the newly formed micro/nanostructures. Overall, $5 \mathrm{~mW} / \mathrm{cm}^{2} \mathrm{UV}$ irradiation mainly benefits the inplane expansion of nanoprotrusions and tends to obtain PI films with protrusions densely distributing on the surface.

Figure 2 presents the SEM images of UV/FAS-treated PI films at the UV intensity of $10 \mathrm{~mW} / \mathrm{cm}^{2}$. The doubled UV intensity was supposed to significantly boost the evolution of the microstructure since higher concentration of active oxygen would be generated [24]. However, SEM images show that the growth of protrusions seemed to be suppressed from the very beginning compared with the $5 \mathrm{~mW} / \mathrm{cm}^{2}$ irradiated samples. During the first 24-hour irradiation, dense and uniform spherical nanoprotrusions with dimension of ca. $50 \mathrm{~nm}$ in diameter formed on the surface and no dendritic protrusion was observed (Figure 2(e)). Instead of rapid growth and expansion in the horizontal direction, dendritic protrusions began to arise only after $48 \mathrm{~h}$ of exposure and became apparent until $72 \mathrm{~h}$. The suppressed growth of protrusions (Figure 2(c)) resulted in larger area of channels compared to $5 \mathrm{~mW} / \mathrm{cm}^{2} \mathrm{UV}$-treated films. As shown in Figures 2(d) and 2(h), further irradiation led to submicron or micron-sized islands that were piled up by the aggregated nanoprotrusions and separated by evenly distributed channels (ca. 200$300 \mathrm{~nm}$ in width). Unlike in the case of $5 \mathrm{~mW} / \mathrm{cm}^{2} \mathrm{UV}$ exposure, wide grooves are not found on the surface, presumably due to the effective release of surface stress through the well-developed channels. In comparison, stronger UV irradiation tends to slow down the horizontal growth of the micro/nanostructures and form island-like nanoprotrusion aggregates as well as well-developed channels in between. 


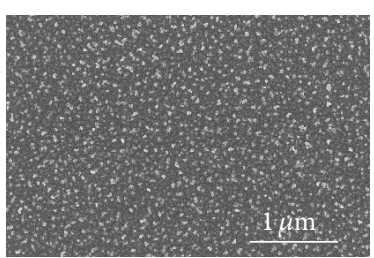

(a)

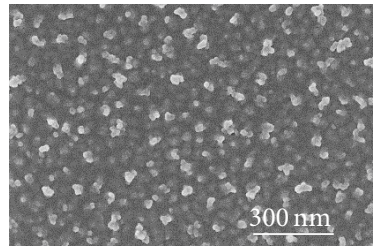

(e)

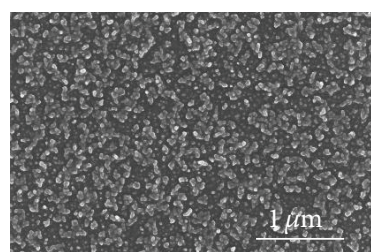

(b)

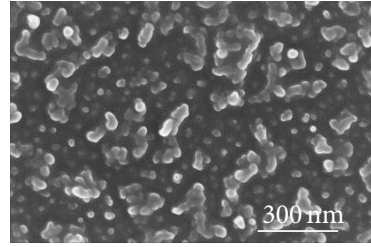

(f)

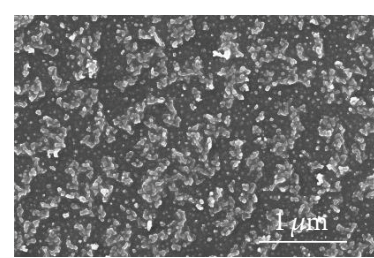

(c)

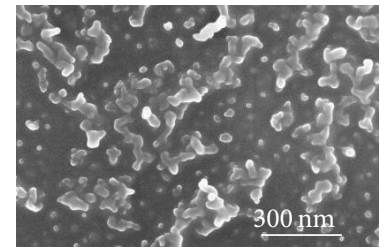

(g)

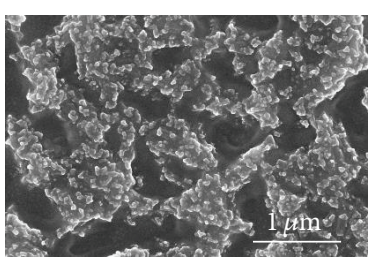

(d)

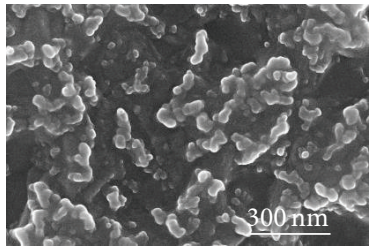

(h)

Figure 3: SEM images of UV $\left(15 \mathrm{~mW} / \mathrm{cm}^{2}\right) / F A S-$ treated PI films. The UV treatment periods are $24 \mathrm{~h}(\mathrm{a}, \mathrm{e}), 48 \mathrm{~h}(\mathrm{~b}, \mathrm{f}), 72 \mathrm{~h}(\mathrm{c}, \mathrm{g}), \mathrm{and} 144 \mathrm{~h}$ $(\mathrm{d}, \mathrm{h})$, respectively.

To further confirm the effects of UV intensity on the evolution of the surface morphology, films treated under $15 \mathrm{~mW} / \mathrm{cm}^{2}$ with various periods were investigated. As shown in Figure 3, the growth of protrusions, especially those in dendritic shape, became much slower when UV intensity increased from 10 to $15 \mathrm{~mW} / \mathrm{cm}^{2}$. Instead, localized growth of the spherical protrusions to the vertical direction of the surface dominated the whole evolution process, leading to the piling-up of the protrusions. The evolution of surface morphology at $15 \mathrm{~mW} / \mathrm{cm}^{2}$ was similar to the polymer surface treated by Ar plasma with shorter exposure time to some extent [25]. In fact, this growth mode is also observed in the $10 \mathrm{~mW} / \mathrm{cm}^{2}$ treated films but not as apparent. When the exposure time was extended to $144 \mathrm{~h}$, interconnecting of the spherical and/or short protrusions also formed island-like aggregates owing to the vertical growth, but with much more and larger groove areas retained in between (Figures $3(\mathrm{~d})$ and 3(h)) than those found in the 5 and $10 \mathrm{~mW} / \mathrm{cm}^{2} \mathrm{UV}$-exposed films. Remarkably, grooves even possess an area nearly equal to that of the protrusion islands in this case.

Therefore, from the above discussion, it can be concluded that the evolution of micro/nanostructures on the surface was governed by distinct protrusion growth modes when UV intensity changed from 5 to $15 \mathrm{~mW} / \mathrm{cm}^{2}$. Increased UV irradiation suppressed the in-plane expansion of protrusions and facilitated the growth in the vertical direction, leading to different surface morphologies.

The evolution of protrusions in horizontal and vertical directions is expected to roughen the surface and thus affect the surface hydrophobicity. While SEM images allow visual assessment of the structural evolution mainly in the horizontal direction, quantified surface roughness values could reflect the growth of protrusions in both horizontal and vertical directions. Therefore, we measured surface roughness of the samples by means of AFM technique.

In Figure 4, root mean square surface roughness (RMS roughness) values at different UV intensities are plotted against the UV irradiation time. Overall, extended irradiation

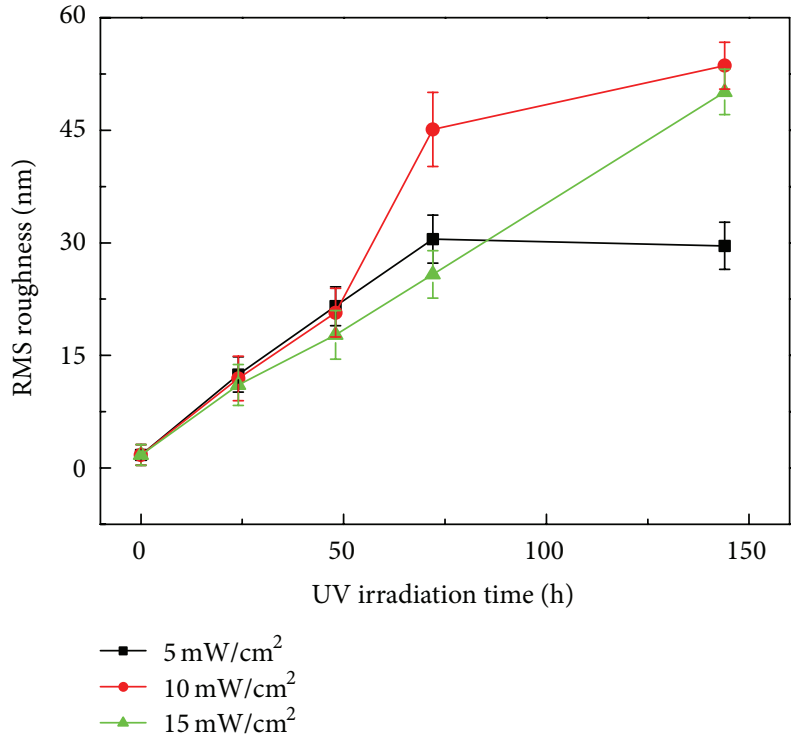

FIGURE 4: RMS roughness plotted against UV radiation time at different UV intensities.

under all UV intensities led to obviously rising trends of the RMS roughness. However, a turning point seemed to appear at $72 \mathrm{~h}$ for $5 \mathrm{~mW} / \mathrm{cm}^{2} \mathrm{UV}$-treated samples. This turning point can be viewed as a result of the fast in-plane expansion of protrusions as described above. In other words, a proper proportion of grooves distributing among the protrusion networks were in favor of the surface roughening, but excessive in-plane expansion of the protrusion areas during the prolonged UV exposure would significantly diminish the groove areas and thus sacrifice the roughness. Then, combining the evolution of the protrusions and that of the grooves, we can speculate that the roughness at ca. $72 \mathrm{~h}$ $(30.6 \mathrm{~nm})$ was the maximum value that PI films could obtain under $5 \mathrm{~mW} / \mathrm{cm}^{2}$ UV irradiation. However, this maximum 


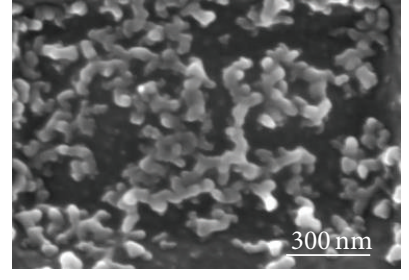

(a)

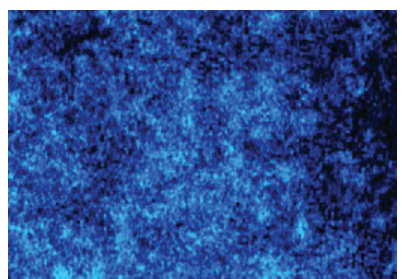

(b)

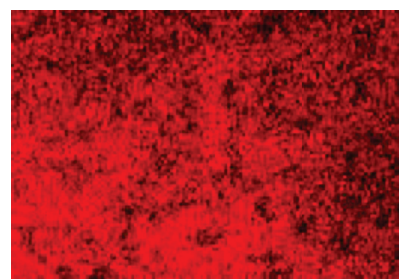

(c)

FIGURE 5: SEM image and corresponding EDS mappings of O (b) and C (c) of UV-treated PI (10 mW/ $\left.\mathrm{cm}^{2}, 72 \mathrm{~h}\right)$. The images were taken at the same position of the sample at the same scale.

roughness was far surpassed by the samples with stronger UV irradiation. Obviously, this can hardly be explained by the suppressed expansion of protrusions in the horizontal directions. Considering the evolution of the microstructures under stronger UV $\left(10\right.$ and $\left.15 \mathrm{~mW} / \mathrm{cm}^{2}\right)$ irradiation described above, we assume that piling-up growth of protrusions in the vertical direction contributed to the surface roughening as well. The fact that roughness values of $10 \mathrm{~mW} / \mathrm{cm}^{2} \mathrm{UV}-$ treated films were larger than those of any other samples also highlighted the combined effects of different protrusion growth modes.

To deepen the understanding of the protrusion evolution and the resultant surface roughness, we have tried to explain the effects of UV intensity from the perspectives of UV photooxidation. Figure 5 demonstrates a typical SEM image and corresponding EDS mappings showing the relation between elemental distributions and surface morphology after UV irradiation $\left(10 \mathrm{~mW} / \mathrm{cm}^{2}\right.$ for $\left.72 \mathrm{~h}\right)$. As shown in Figure 5, protrusion areas are rich in O, yet poor in C. Given the fact that highly volatile and mobile low-molecular-weight (LMW) polymer chains would form as a result of the UV photooxidation induced chain scission [26], the protrusions are deemed to stem from the migration and redeposition of the oxidized polymer chains during UV irradiation [12]. Therefore, we think that UV intensity affected the protrusion evolution by changing the process of polymer chain scission and redeposition. Stronger UV irradiation would lead to higher photooxidation degree of polymer with shorter chains due to more concentrated active oxygen [24]. These oxidized polymers were more likely to volatilize and leave rather than redeposit on the surface, thus leading to the lower expansion rate of the protrusions. Another reason for the suppressed inplane growth of the protrusions is the mismatch of interface energy between highly oxidized polymer chains and the newly formed PI surface, which was unfavorable for the redeposition on less oxidized areas, especially the groove areas. This can also explain why the protrusions preferred to grow in a piling-up mode when UV intensity increased.

3.2. Hydrophobicity. According to the Wenzel equation [27], surface roughening would further enhance the antiwetting capability if the smooth surface exhibits a hydrophobic nature. The hydrophobicity is strongly dependent on the RMS roughness of the surface. Therefore, it can be speculated that the hydrophobicity of the PI samples would change with the

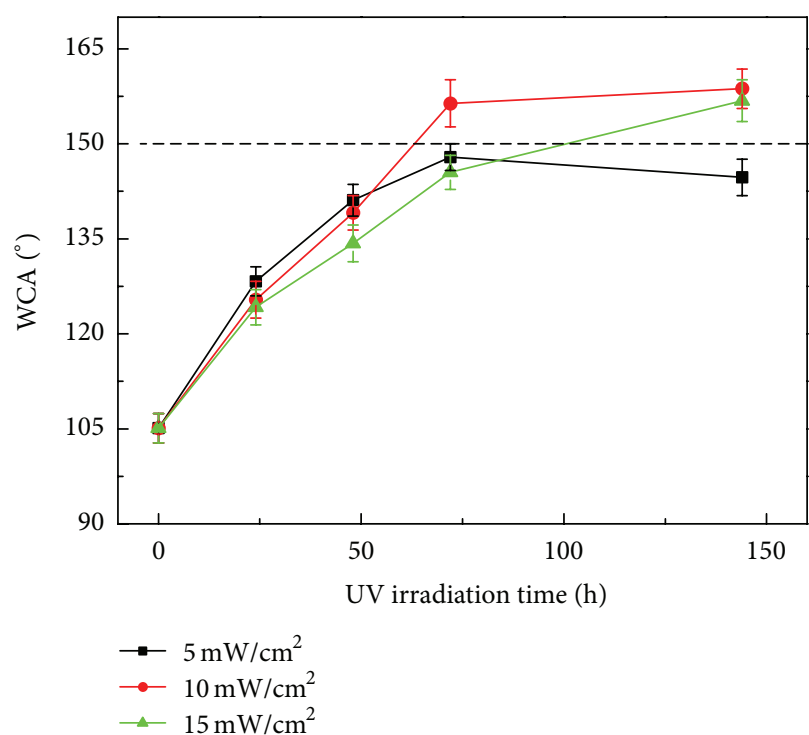

FIGURE 6: WCA plotted against UV radiation time at different UV intensities.

UV exposure period as well as UV intensity. To quantitatively confirm this relationship, WCA was used in this study to evaluate the hydrophobicity of the PI films.

The WCA against UV irradiation time at different UV intensities are plotted in Figure 6. The evolution of WCA is substantially consistent with the surface roughness in Figure 5. At the intensity of $5 \mathrm{~mW} / \mathrm{cm}^{2}$, WCA increased from $105.1^{\circ}$ to $148.1^{\circ}$ with irradiation time extended from $0 \mathrm{~h}$ to $72 \mathrm{~h}$ and showed a relatively low hydrophobicity at $144 \mathrm{~h}$ with WCA of $144.7^{\circ}$. The WCA value of $5 \mathrm{~mW} / \mathrm{cm}^{2}$ at $144 \mathrm{~h}$ decreased as a result of the excessive in-plane expansion of the protrusion areas and the consequent surface roughness decrease as depicted in Figure 5. In contrast, the WCA increased continuously to $159.2^{\circ}$ at the intensity of $10 \mathrm{~mW} / \mathrm{cm}^{2}$ and to $156.9^{\circ}$ at $15 \mathrm{~mW} / \mathrm{cm}^{2}$ when UV exposure time was prolonged to $144 \mathrm{~h}$. Eventually, superhydrophobic surfaces with WCA above $150^{\circ}$ could be achieved after $72 \mathrm{~h}$ of irradiation at the intensity of $10 \mathrm{~mW} / \mathrm{cm}^{2}$ or after $144 \mathrm{~h}$ at $15 \mathrm{~mW} / \mathrm{cm}^{2}$. As another important characteristic parameter, the sliding angle was further investigated for the superhydrophobic samples. As shown in Figure 7, water drops $(10 \mu \mathrm{L})$ were found to readily roll off all the superhydrophobic PI 


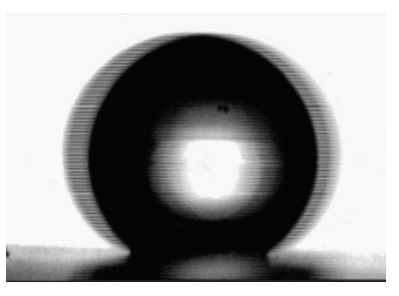

(a)

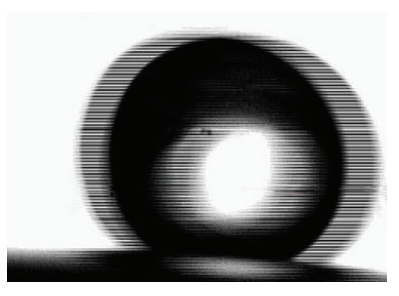

(b)

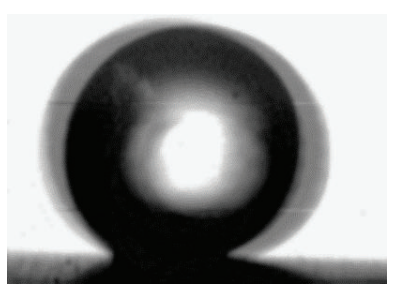

(c)

Figure 7: Water droplets $(10 \mu \mathrm{L})$ sliding on slightly tilted surfaces. The UV treatment parameters are $72 \mathrm{~h}$ at $10 \mathrm{~mW} / \mathrm{cm}^{2}(\mathrm{a}), 144 \mathrm{~h}$ at $10 \mathrm{~mW} / \mathrm{cm}^{2}$ (b), and $144 \mathrm{~h}$ at $15 \mathrm{~mW} / \mathrm{cm}^{2}$ (c), respectively. These were critical images obtained by slowly tilting the sample stage until the $10 \mu \mathrm{L}$ water droplet started moving using a digital video camera.

films with the tilted angle below $5^{\circ}$. These data strongly support that superhydrophobicity could be obtained by both $10 \mathrm{~mW} / \mathrm{cm}^{2}$ and $15 \mathrm{~mW} / \mathrm{cm}^{2}$ UV irradiation.

It should also be stressed that rational choosing of UV intensity is very important for the fabrication of superhydrophobic PI materials. Either too low or too high UV intensity is adverse to superhydrophobicity. In a relatively short period $(72 \mathrm{~h})$, a medium UV intensity of $10 \mathrm{~mW} / \mathrm{cm}^{2}$ could afford the films WCA larger than $150^{\circ}$ and sliding angle below $5^{\circ}$. In contrast, $5 \mathrm{~mW} / \mathrm{cm}^{2}$ UV irradiation failed to achieve superhydrophobicity, and a longer time was proven to be required (up to $144 \mathrm{~h}$ ) for $15 \mathrm{~mW} / \mathrm{cm}^{2}$. Considering the efficiency and costs, UV photooxidation under $10 \mathrm{~mW} / \mathrm{cm}^{2}$ for $72 \mathrm{~h}$ is an optimized choice to obtain superhydrophobic PI films.

It is worth noting that the trend discussed above was different from the case in which laser ablation or plasma treatment was used to engineer the surface structures of polymers [20-22]. Generally, the surface roughness and hydrophobicity of PI surfaces treated by shaped laser increased when intensity or exposure time was enhanced [11,20], which was quite different from the evolution modes of surface morphology and corresponding surface roughness when UV intensity increased in this work. The difference could be explained by different surface dynamics. Under UV irradiation of polymers in air, the polymer surfaces undergo chemical modification (photooxidation and induced hydrolysis) and induced degradation, crosslinking, and redeposition. Whereas for the laser ablation its energy is deposited in a short period, ablation dominated the morphological change while the crosslinking and redeposition could be neglected for laser-treated PI surfaces [28].

\section{Conclusions}

In summary, we investigated the effects of UV intensity on the PI surface morphology and wetting performance, following the development of UV photooxidation as a method to prepare hydrophobic PI in combination with FAS modification. Surface morphology was found to present different evolutionary patterns against UV irradiation time when UV intensity varied. $5 \mathrm{~mW} / \mathrm{cm}^{2} \mathrm{UV}$ intensity facilitated in-plane expansion of dendritic protrusions while 10 and $15 \mathrm{~mW} / \mathrm{cm}^{2}$ UV intensity encouraged localized growth of protrusions in a piling-up manner. Surface roughness and hydrophobicity maximized at the intensity of $10 \mathrm{~mW} / \mathrm{cm}^{2}$. The mechanism that enhanced degree of photooxidation under stronger UV intensity suppressed the horizontal expansion and boosted the preferred vertical growth of protrusions was proposed to explain the changes in evolutionary patterns of surface structure. Though superhydrophobicity (WCA larger than $150^{\circ}$ ) can be achieved at UV intensity not less than $10 \mathrm{~mW} / \mathrm{cm}^{2}$, a longer time was proven to be required (up to $144 \mathrm{~h}$ for $15 \mathrm{~mW} / \mathrm{cm}^{2}$ ). Considering the efficiency and costs, UV photooxidation under $10 \mathrm{~mW} / \mathrm{cm}^{2}$ for $72 \mathrm{~h}$ is an optimized choice to obtain superhydrophobic PI films. We expect that this work would further promote the application of UV method in hydrophobicity control of PI materials.

\section{Conflict of Interests}

The authors declare that there is no conflict of interests regarding the publication of this paper.

\section{References}

[1] K. Liu, Y. Tian, and L. Jiang, "Bio-inspired superoleophobic and smart materials: design, fabrication, and application," Progress in Materials Science, vol. 58, no. 4, pp. 503-564, 2013.

[2] K. Liu and L. Jiang, "Bio-inspired self-cleaning surfaces," Annual Review of Materials Research, vol. 42, pp. 231-263, 2012.

[3] H. Li, M. Qu, Z. Sun, J. He, and A. Zhou, "Facile fabrication of a hierarchical superhydrophobic coating with aluminate coupling agent modified kaolin," Journal of Nanomaterials, vol. 2013, Article ID 497216, 5 pages, 2013.

[4] T. Darmanin, E. T. de Givenchy, S. Amigoni, and F. Guittard, "Superhydrophobic surfaces by electrochemical processes," Advanced Materials, vol. 25, no. 10, pp. 1378-1394, 2013.

[5] M. Wolfs, T. Darmanin, and F. Guittard, "Superhydrophobic fibrous polymers," Polymer Reviews, vol. 53, no. 3, pp. 460-505, 2013.

[6] N. Wang, F. Guo, J. Wu, Y. Zhao, and L. Jiang, "Variable responsive wettability films via electrospinning induced by solvents," Journal of Nanomaterials, vol. 2014, Article ID 817418, 7 pages, 2014.

[7] G. Gong, J. Wu, J. Liu, N. Sun, Y. Zhao, and L. Jiang, "Bioinspired adhesive superhydrophobic polyimide mat with high thermal stability," Journal of Materials Chemistry, vol. 22, no. 17, pp. 8257-8262, 2012. 
[8] M. K. Ghosh and K. L. Mittal, Polyimides: Fundamentals and Applications, CRC Press, 1996.

[9] Y. Zhu, P. Zhao, X. Cai, W.-D. Meng, and F.-L. Qing, "Synthesis and characterization of novel fluorinated polyimides derived from bis[4-( $4^{\prime}$-aminophenoxy)phenyl]-3,5-bis (trifluoromethyl)phenyl phosphine oxide," Polymer, vol. 48, no. 11, pp. 3116-3124, 2007.

[10] S. Mu, D. Wu, S. Qi, and Z. Wu, "Preparation of polyimide/Zinc oxide nanocomposite films via an ion-exchange technique and their photoluminescence properties," Journal of Nanomaterials, vol. 2011, Article ID 950832, 10 pages, 2011.

[11] C. J. Wohl, M. A. Belcher, L. Chen, and J. W. Connell, "Laser ablative patterning of copoly(imide siloxane)s generating superhydrophobic surfaces," Langmuir, vol. 26, no. 13, pp. 1146911478, 2010.

[12] H. Y. Gu, Z. Y. Qi, W. Wu, Y. Zeng, and L. X. Song, "Superhydrophobic polyimide films with high thermal endurance via UV photo-oxidation," Express Polymer Letters, vol. 8, no. 8, pp. 588595, 2014.

[13] T. Nishino, M. Meguro, K. Nakamae, M. Matsushita, and Y. Ueda, "The lowest surface free energy based on -CF3 alignment," Langmuir, vol. 15, no. 13, pp. 4321-4323, 1999.

[14] K.-C. Chang, H.-I. Lu, C.-W. Peng et al., "Nanocasting technique to prepare lotus-leaf-like superhydrophobic electroactive polyimide as advanced anticorrosive coatings," ACS Applied Materials \& Interfaces, vol. 5, no. 4, pp. 1460-1467, 2013.

[15] Y. Zhao, M. Li, Q. Lu, and Z. Shi, "Superhydrophobic polyimide films with a hierarchical topography: combined replica molding and layer-by-layer assembly," Langmuir, vol. 24, no. 21, pp. 12651-12657, 2008.

[16] S. Zhu, Y. Li, J. Zhang et al., "Biomimetic polyimide nanotube arrays with slippery or sticky superhydrophobicity," Journal of Colloid and Interface Science, vol. 344, no. 2, pp. 541-546, 2010.

[17] K. Liu, J. Du, J. Wu, and L. Jiang, "Superhydrophobic gecko feet with high adhesive forces towards water and their bio-inspired materials," Nanoscale, vol. 4, no. 3, pp. 768-772, 2012.

[18] C.-J. Weng, Y.-S. Jhuo, C.-H. Chang et al., "A smart surface prepared using the switchable superhydrophobicity of neat electrospun intrinsically electroactive polyimide fiber mats," Soft Matter, vol. 7, no. 21, pp. 10313-10318, 2011.

[19] J. Y. Park, K. O. Oh, J. C. Won, H. Han, H. M. Jung, and Y. S. Kim, "Facile fabrication of superhydrophobic coatings with polyimide particles using a reactive electrospraying process," Journal of Materials Chemistry, vol. 22, no. 31, pp. 16005-16010, 2012.

[20] V. Oliveira, B. Nunes, and R. Vilar, "Wetting response of KrF laser ablated polyimide surfaces," Nuclear Instruments and Methods in Physics Research, Section B: Beam Interactions with Materials and Atoms, vol. 268, no. 10, pp. 1626-1630, 2010.

[21] G. Scheen, K. Ziouche, Z. Bougrioua, P. Godts, D. Leclercq, and T. Lasri, "Simultaneous fabrication of superhydrophobic and superhydrophilic polyimide surfaces with low hysteresis," Langmuir, vol. 27, no. 10, pp. 6490-6495, 2011.

[22] H. C. Barshilia, A. Ananth, N. Gupta, and C. Anandan, "Superhydrophobic nanostructured Kapton surfaces fabricated through $\mathrm{Ar}+\mathrm{O}_{2}$ plasma treatment: effects of different environments on wetting behaviour," Applied Surface Science, vol. 268, pp. 464-471, 2013.

[23] C. J. Wohl, M. A. Belcher, S. Ghose, and J. W. Connell, "Modification of the surface properties of polyimide films using polyhedral oligomeric silsesquioxane deposition and oxygen plasma exposure," Applied Surface Science, vol. 255, no. 18, pp. 8135-8144, 2009.

[24] M. L. Sham, J. Li, P. C. Ma, and J.-K. Kim, "Cleaning and functionalization of polymer surfaces and nanoscale carbon fillers by uv/ozone treatment: a review," Journal of Composite Materials, vol. 43, no. 14, pp. 1537-1564, 2009.

[25] J. Yun, T.-S. Bae, J.-D. Kwon, S. Lee, and G.-H. Lee, "Antireflective silica nanoparticle array directly deposited on flexible polymer substrates by chemical vapor deposition," Nanoscale, vol. 4, no. 22, pp. 7221-7230, 2012.

[26] U. Sener, B. Parekh, A. Entenberg, T. Debies, and G. A. Takacs, "Surface modification of poly(biphenyl dianhydridepara-phenylene diamine) (BPDA-PDA) polyimide by UV photo-oxidation," Journal of Adhesion Science and Technology, vol. 20, no. 4, pp. 319-334, 2006.

[27] R. N. Wenzel, "Resistance of solid surfaces to wetting by water," Industrial \& Engineering Chemistry, vol. 28, no. 8, pp. 988-994, 1936.

[28] H. Hiraoka and S. Lazare, "Surface modifications of Kapton and cured polyimide films by ArF excimer laser: applications to imagewise wetting and metallization," Applied Surface Science, vol. 46, no. 1-4, pp. 264-271, 1990. 

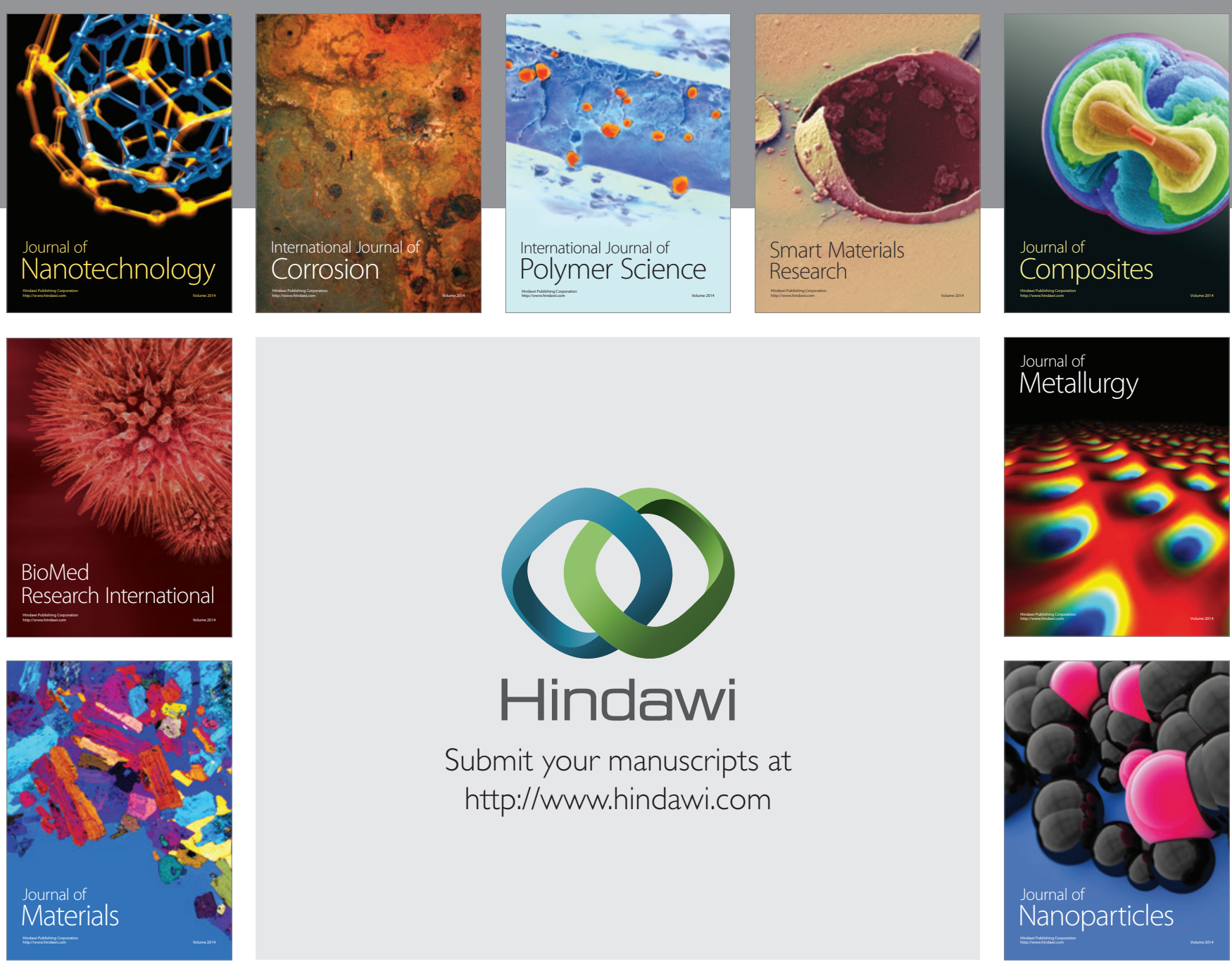

Submit your manuscripts at http://www.hindawi.com
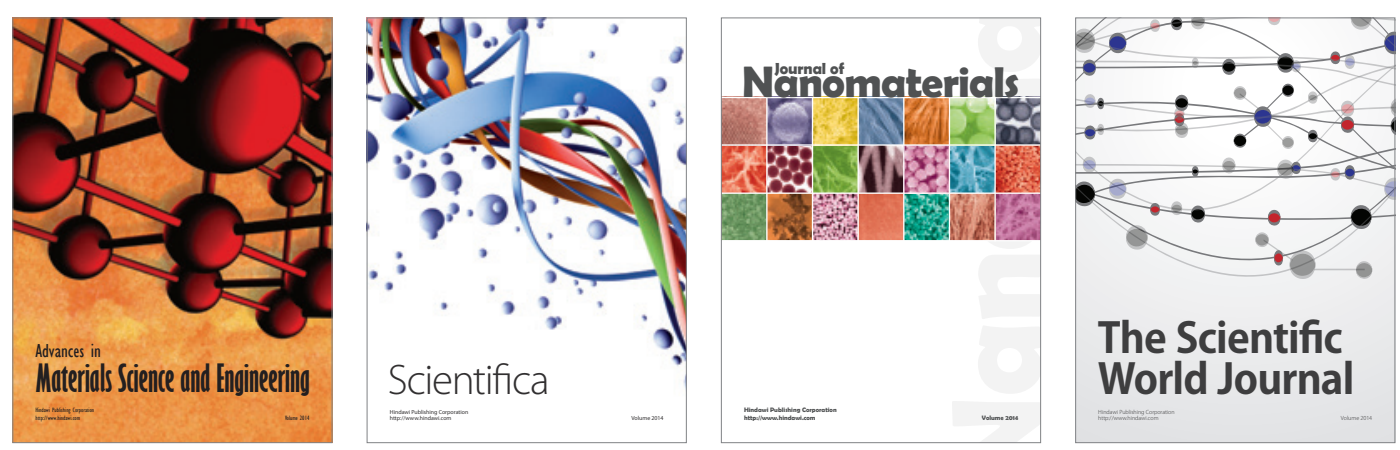

\section{The Scientific World Journal}
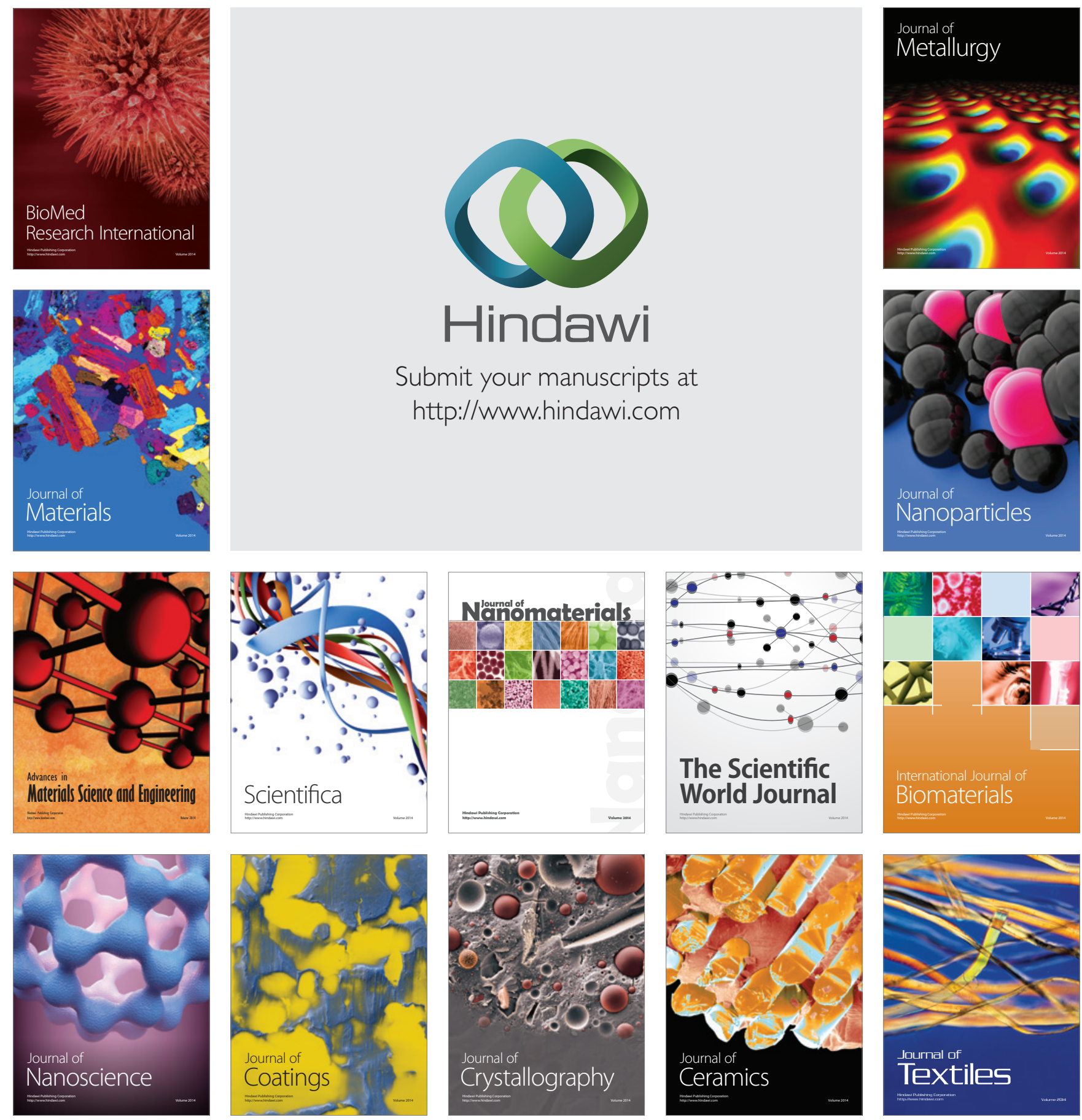